\title{
Pharmacovigilance and Pharmacoepidemiology
}

EDELWEISS PUBLICATIDNS IPEN ACCESS

ISSN: 2638-8235

\section{Letter to Editor \\ Gull Alpha Power of the Chen Type}

\section{Clement Boateng Ampadu*}

Affiliation: 31 Carrolton Road, Boston, MA 02132-6303, USA

*Corresponding author: Clement Boateng Ampadu, Department of Biostatistics, USA, Tel: +1-6174697268, E-mail:

drampadu@hotmail.com

Citation: Ampadu BC. Gull alpha power of the chen type (2020) Pharmacovigil and Pharmacoepi 3: 16-17.

Received: Oct 28, 2020

Accepted: Dec 05, 2020

Published: Dec 11, 2020

Copyright: (C) 2020 Ampadu BC. This is an open-access article distributed under the terms of the Creative Commons Attribution License, which permits unrestricted use, distribution, and reproduction in any medium, provided the original author and source are credited.

\section{Abstract}

In they introduced the Chen distribution, and in they extended the distribution to include its "normalized version". In this paper, we introduce a variant of the Gull Alpha Power distribution by modifying Chen-G of and show the new family is good in fitting real life data.

Keywords: Chen distribution, Chen-G distribution, Gull alpha power distribution.

\section{Introduction to the New Family}

In 2000, the following distribution was introduced in [1] as the Chen distribution, $\mathbf{F}(\mathbf{t})=\mathbf{1}-\mathrm{e}^{\lambda\left(1-e^{t \beta}\right)}$, where $\lambda, \beta, \mathrm{t}>0$. The two parameter Chen distribution has the ability to model bathtub shaped failure rate functions; it however lacks a scale parameter. The normalized version was introduced in [2] with the following CDF,

$$
\mathrm{F}(\mathrm{x})=\int_{0}^{G(\chi)} \mathrm{f}(\mathrm{t}) \mathrm{dt}=\mathrm{A}\left[1-\mathrm{e}^{\lambda}\left(1-\mathrm{e}^{G(x) B}\right)\right]
$$

Where $\lambda, \beta>0, x 2 \mathrm{R}$ and $A=\frac{1}{1-e^{\lambda(1-e)}}$ is a normalizing constant. Based on the structure of the Gull Alpha Power CDF [3] $\frac{\alpha^{F}(y)}{\alpha^{F(y)}}$ for $\alpha>1$, we modify Chen-G [2] to introduce the following.

\section{Definition}

A random variable $\mathrm{J}$ will be called Gull Alpha Power distributed with respect to Chen if its $\mathrm{CDF}$ is given by

$$
\mathrm{F}(\mathrm{x} ; \mathrm{a}, \mathrm{b}, \xi)=\frac{\left(e^{(1-e)^{a}}+1\right) G(x ; \xi)}{e^{a\left(1-e^{G(x ; \xi) b}\right)+1}}
$$

Where $\mathrm{a}, \mathrm{b}>0, \mathrm{G}$ is some baseline distribution with parameter vector $\xi$, and $\chi \in \mathbb{R}$. The new distribution is a good fit to real life data as shown in the next section.

\section{Practical Illustration}

We assume the baseline distribution is Normal with the following $\mathrm{CDF} \quad \mathrm{G}(\mathrm{x} ; \xi)=\frac{1}{2} \operatorname{erfc} \frac{c-x}{\sqrt{2 d}}$ where erfc is the complementary error function. Thus, from the above definition, we have the following.

\section{Proposition}

The CDF of the Gull Alpha Power Normal distribution of the Chen Type is given by

$$
\mathrm{F}(\mathrm{x} ; \mathrm{a}, \mathrm{b}, \mathrm{c}, \mathrm{d})=\frac{\left(e^{(1-e) a}+1\right) \operatorname{erfc}\left(\frac{c-x}{\sqrt{2 b}}\right)}{2\left(e^{a\left((1-e)^{2-b} \operatorname{erfc}\left(\frac{c-x}{\sqrt{2}}\right)^{b}\right)}+1\right)}
$$

Obviously, the PDF can be obtained by differentiating the CDF above.

\section{Notation}

We write $\mathbf{J} \sim$ GAPANC(c,d,a,b) if $\mathbf{J}$ is a Gull Alpha Power Normal random variable of the Chen type

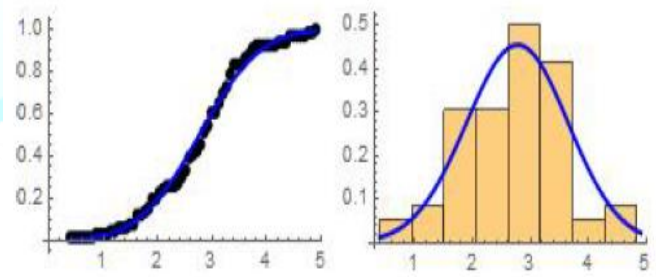

Figure 1: The CDF and PDF of GAPANC $(2.71318,0.884185$, $0.160665,0.457947)$ fitted to the empirical distribution and histogram of the lifetime of 50 devices data [4].

\section{Open Problem}

It is an open problem to obtain properties and applications of this new class of statistical distributions. The author invites readers to tackle this open problem. 


\section{References}

1. Chen Z. A new two-parameter lifetime distribution with bathtub shape or increasing failure rate function (2000) Statistics and Probability Letters 49: 155-161. https://doi.org/10.1016/S0167-7152(00)00044-4

2. Anzagra L, Sarpong $\mathrm{S}$ and Nasiru S. Chen-G class of distributions (2020) Cogent Mathematics and Statistics 7. https://doi.org/10.1080/25742558.2020.1721401

3. Ijaz M, Asim SM, Alamgir, Farooq M, Khan SA, et al. A gull alpha power weibull distribution with applications to real and $\begin{array}{lllll}\text { simulated data (2020) PLoS ONE } 15 . & \end{array}$ https://doi.org/10.1371/journal.pone.0233080

4. Aarset MV. How to identify bathtub hazard rate (1987) IEEE Transactions on Reliability 36: 106-108. 